\title{
More power to you: Simple power calculations for treatment effects with one degree of freedom
}

\author{
JAMIE I. D. CAMPBELL and VALERIE A. THOMPSON \\ University of Saskatchewan, Saskatoon, Saskatchewan, Canada
}

\begin{abstract}
Although numerous computer programs for statisticalpower analysis are available, power is an underused aspect of experimental analysis, perhaps because of the perceived difficulty of performing the necessary calculations or because existing computer software can be expensive or complicated to learn. For single-degree-of-freedomtests, however, it is possible to calculate power in a straightforward manner, using the $t$ distribution. Because these calculations are based on $t$, they use easily understood and readily available quantities. These calculations can be performed with a desk calculator; we also present a simple-to-use program called MorePower that will perform the necessary calculations. The straightforward nature of the calculations potentially will enable more researchers to consider issues of power when planning and reporting their experiments.
\end{abstract}

There are many sophisticated computer programs available for power analysis, but these may be expensive, complicated to learn, or limited in their application (Thomas \& Krebs, 1997). Here, we present simple formulas for calculating power and related statistics for any analysis of variance (ANOVA) test with one degree of freedom. We explain how to do the calculations by hand, as well as by using a simple-to-use freeware program for Microsoft Windows, called MorePower (http://duke.usask.ca/ campbelj/ work/MorePowerx.html). Our formulas and program have several distinctive features. First, because they are based on the familiar and relatively simple $t$ test, the formulas and program are easy to use and understand; nonetheless, they apply to any single-degree-of-freedom test (main effect or interaction) from within-subjects, betweensubjects, or mixed designs of arbitrary complexity (assuming equal $n$ s for between-subjects factors). Second, effect size is estimated (or provided by the user) in the original units of measurement, rather than in less intuitive variancebased ratios or small-medium-large approximations [the MorePower program also calculates $\eta^{2}$, however; i.e., MST/ $(M S T+M S E * d f)]$. Third, to specify effect size, one may enter either $M S T$ or the difference in original units; to specify error variance, one may enter either $M S E$ or the variance of the difference. Thus, given the $F$-ratio and $M S E$ provided in most research reports, one can easily

This work was funded by the Natural Sciences and Engineering Research Council of Canada. We thank Raymond Gunter, Lorin Elias, Deborah Saucier, and Eyvind Ohm for their feedback and suggestions on the MorePower program. The authors contributed equally to this paper. Address correspondence to J. I. D. Campbell or V. A. Thompson, Department of Psychology, University of Saskatchewan, 9 Campus Drive, Saskatoon, SK, S7N 5A5 Canada (e-mail: jamie.campbell@usask.ca or valerie.thompson@usask.ca). compute observed power and effect size for any ANOVA effect with one degree of freedom. This makes it a trivial task to assess the power associated with published null effects or to use previous results to estimate the required effect size or sample size for planned experiments.

\section{Calculating Power for the \\ Between-Subjects $t$ Test}

Our power analysis extends the approach developed by Cochran and Cox (1957) for the between-subjects $t$ test (also, see Hays, 1994, for an analogous derivation for $z$ ). Ordinarily, one rejects the null hypothesis when the observed difference between means $\left(d_{\mathrm{c}}\right)$ is large enough such that $t$ exceeds the value set a priori to represent the Type I error rate $\left(t_{\alpha}\right)$ :

$$
d_{\mathrm{c}}>t_{\alpha} \sqrt{\frac{2 \cdot s_{d}^{2}}{n_{\mathrm{t}}}} .
$$

In Equation 1, $s_{d}^{2}=$ variance of the difference between means and $n_{\mathrm{t}}=$ number of observations per treatment (equal $n$ s assumed). Power is the probability of drawing a sample in which $d_{\text {c }}$ satisfies the above requirement from a sampling distribution whose mean $\left(d_{\beta}\right)$ is greater than 0 ; power, therefore, is the probability that $t>t_{\beta}$ :

$$
t_{\beta}=\frac{d_{\mathrm{c}}-d_{\beta}}{\sqrt{\frac{2 \cdot s_{d}^{2}}{n_{\mathrm{t}}}}} .
$$

For our analysis, we combined Equations 1 and 2 and then solved for power (probability $t>t_{\beta}$ ), effect size $\left(d_{\beta}\right)$, and sample size $\left(n_{\mathrm{t}}\right)$. 
Power:

$$
t_{\beta}=t_{\alpha}-\frac{d_{\beta}}{\sqrt{\frac{s_{d}^{2}}{n_{\mathrm{t}}}}}
$$

Effect Size:

$$
d_{\beta}=\sqrt{\frac{\left(t_{\alpha}-t_{\beta}\right)^{2} \cdot s_{d}^{2}}{n_{\mathrm{t}}}}
$$

Sample Size:

$$
n_{t}=\frac{\left(t_{\alpha}-t_{\beta}\right)^{2} \cdot s_{d}^{2}}{d_{\beta}^{2}} .
$$

Extension to multifactor designs. We extend this analysis to multifactor ANOVA designs, including betweensubjects, within-subjects, and mixed designs. This extension is possible because $s_{d}^{2}$ can be derived from $M S E$ (see below) and because $F$ and $t^{2}$ are equivalent for any $2^{k}$ effect in a multifactor design. That is, any $F$ test with one degree of freedom in the numerator corresponds to a pairwise comparison (e.g., a $2 \times 2$ interaction corresponds to a pairwise comparison of the difference of differences), even when the $2^{k}$ effect is embedded in a design that includes factors with more than two levels.

\section{Quantities Needed for Calculations}

Power, sample size, and effect size can be calculated by hand using Equations 3, 4, and 5, or by using the MorePower program, which is described in the next section. In either case, the researcher needs to have an estimate of error variance ( $s_{d}^{2}$ or $M S E$ ) and a value for $\alpha$, as well as two of the remaining three variables: sample size $\left(n_{\mathrm{t}}\right)$, effect size $\left(d_{\beta}\right)$, or power (one can calculate the third, given values for the other two).

Computing $s_{d}^{2}$. One can estimate $s_{d}^{2}$ either from an estimate of individual cell variances or from MSE (MorePower will calculate $s_{d}^{2}$, given a value of $M S E$ ). Equation 6 calculates $s_{d}^{2}$ from individual cell variances. For betweensubjects designs, the intercell correlations are all zero; thus, the value of the second half of Equation 6 is reduced to zero:

$$
s_{d}^{2}=s_{1}^{2}+s_{2}^{2}-2 r_{12} s_{1} s_{2} .
$$

Equation 7 computes $s_{d}^{2}$ from $M S E$ :

$$
s_{d}^{2}=M S E \cdot 2^{B} \cdot \frac{2^{W \cdot 2}}{y} .
$$

In this equation, $B$ is the number of two-level betweensubjects factors in the relevant test, $W$ is the number of two-level within-subjects factors in the relevant test, and $y$ is the total number of within-subjects cells in the experiment. This equation can be used to calculate $s_{d}^{2}$ for any comparison, including those having both between- and within-subjects factors. Note that when the effect of interest involves only between-subjects or only withinsubjects factors, Equation 7 can be simplified to Equations $7 \mathrm{a}$ and $7 \mathrm{~b}$, respectively:

$$
s_{d}^{2}=M S E \cdot 2^{B}
$$

and

$$
s_{d}^{2}=M S E \cdot \frac{2^{W \cdot 2}}{y} .
$$

Calculating $\boldsymbol{d}_{\beta}$ (effect size). The quantity $d_{\beta}$ refers to the size of the difference one is interested in and is equal to the absolute difference between treatment conditions. For pairwise comparisons and main effects, therefore, $d_{\beta}$ equals the absolute value of the difference between the means for the two levels of the relevant factor, averaged over the levels of the other factors. For $2 \times 2$ interactions, $d_{\beta}$ is the absolute value of the difference of differences, $\left(M_{11}-M_{12}\right)-\left(M_{21}-M_{22}\right)$ (again, averaged over the levels of other factors), and for $2 \times 2 \times 2$ interactions, it is the absolute value of the difference between the difference of differences $\left[\left(M_{111}-M_{112}\right)-\left(M_{121}-M_{122}\right)\right]-$ $\left[\left(M_{211}-M_{212}\right)-\left(M_{221}-M_{222}\right)\right]$ and so on. For example, if $M_{11}, M_{12}, M_{21}$, and $M_{22}$ were equal to 50, 30, 15, and 5 , then $d_{\beta}$ for the $2 \times 2$ interaction would equal (50$30)-(15-5)$, or 10 .

The observed effect size for a specific main or interaction effect can also be calculated using MST from the corresponding ANOVA test. Specifically,

$$
d_{\beta}=\sqrt{\left(M S T \cdot 2^{B} \cdot \frac{2^{W \cdot 2}}{y}\right) / n_{\mathrm{t}}},
$$

where $B, W$, and $y$ are defined as above. The MorePower program allows the user to enter a value for either $d_{\beta}$ or $M S T$ to estimate power or effect size; $d_{\beta}$ must be supplied to estimate sample size.

Calculating $\boldsymbol{n}_{\mathbf{t}}$. This quantity is the number of observations that contribute to each treatment level in the effect of interest. Equal $n$ s are assumed for between-subjects factors. For $2^{k}$ effects composed entirely of within-subjects factors (i.e., no between-subjects factors), $n_{\mathrm{t}}$ is equal to the total $N$. If the $2^{k}$ effect of interest includes one betweensubjects factor, then $n_{\mathrm{t}}$ is the number of observations contributing to each level of the between-subjects factor $\left(n_{\mathrm{t}}=\right.$ $N / 2$ ); if the effect includes two between-subjects factors (e.g., a $2 \times 2 \times 2$ interaction involving two betweensubjects and one within-subjects factor), then $n_{\mathrm{t}}$ is the number of observations in each of the four between-subjects cells $\left(n_{\mathrm{t}}=N / 4\right)$, and so on.

\section{Calculating Power, Effect Size, and Sample Size With MorePower}

The MorePower program was created using VisualBasic 5.0 for Microsoft Windows and should run under any version of the Windows operating system when installed with 
the self-extracting MPSetup.exe program available at http://duke.usask.ca/ campbelj/work/MorePowerx.html. MPSetup installs (if necessary) all the Windows system and VisualBasic 5.0 files (5.4 MB) required for MorePower, but we found that most of the $\approx 50$ computers tested already had the necessary Windows components. Consequently, usually one requires only the MorePower.exe file (100K), which can be downloaded separately. The program requires a screen resolution of $800 \times 600$ pixels or higher.

The MorePower program interface is depicted in Figure 1. Point and click the mouse on the appropriate label in the Estimate panel to select the quantity one wishes to estimate: power, $d_{\beta}$ (Effect Size on the program interface), or $n_{\mathrm{t}}(n /$ treatment on the program interface). This clears and highlights the window for that quantity and moves the cursor to set alpha, which is .05 by default. Set alpha to $\alpha * 2$ for a one-tailed test. Pressing Enter on one's keyboard moves the cursor to the next appropriate box, or one may move between boxes by pointing and clicking the mouse.

In the Effect Size panel, point and click the mouse on the label or data entry field to select $M S T$ or $d_{\beta} ; d_{\beta}$ must be $>0$ to estimate $n_{\mathrm{t}}$. Similarly, in the Error Variance panel, point and click the mouse to select $M S E$ or $s_{d}^{2}$.

One must also specify information in the Effect and Design panel (see Figure 1). Here, one specifies the effect of interest and the design of the total experiment. Figure 1 illustrates the case in which the researcher is interested in estimating the sample size required (i.e., $n /$ treatment) for a $2 \times 2$ mixed interaction (i.e., one between- and one withinsubjects factors) in the context of a $2 \times 2 \times 2 \times 2$ experiment (two within- and two between-subjects factors).

Effect Betwn and Effect Within refer to the number of two-level between- and within-subjects factors in the effect of interest. In the case of the $2 \times 2$ mixed interaction described above, these values are 1 and 1 , respectively (i.e., there is one within- and one between-subjects factor in the interaction), as is illustrated in Figure 1. For a withinsubjects main effect, the values would be 0 (or blank) and 1 ; for the interaction of two between-subjects variables with one within-subjects variable, these values would be 2 and 1 , and so on.

Betwn Factors and Within Factors specify the design of the complete experiment. Specifically, the user must enter the number of levels associated with each of the betweenand within-subjects factors in the complete design, including those involving three or more levels. For example, if the experiment consists of two between- and two withinsubjects factors, enter 22 for Betwn Factors and 22 for Within Factors, as is illustrated in Figure 1. For a $2 \times$ $2 \times 3$ within-subjects design, the sequence 223 (or 232 or 322) would be entered for Within Factors and Betwn Factors would be set to 0 or left blank. For a betweensubjects $t$ test, enter 2 for Betwn Factors and 0 (or blank) for Within Factors.

Clicking the Calculate button or pressing Enter on the keyboard with the cursor in the Within Factors window initiates calculation based on the data entered. If the Calculate button is pressed with missing or incorrect quanti-

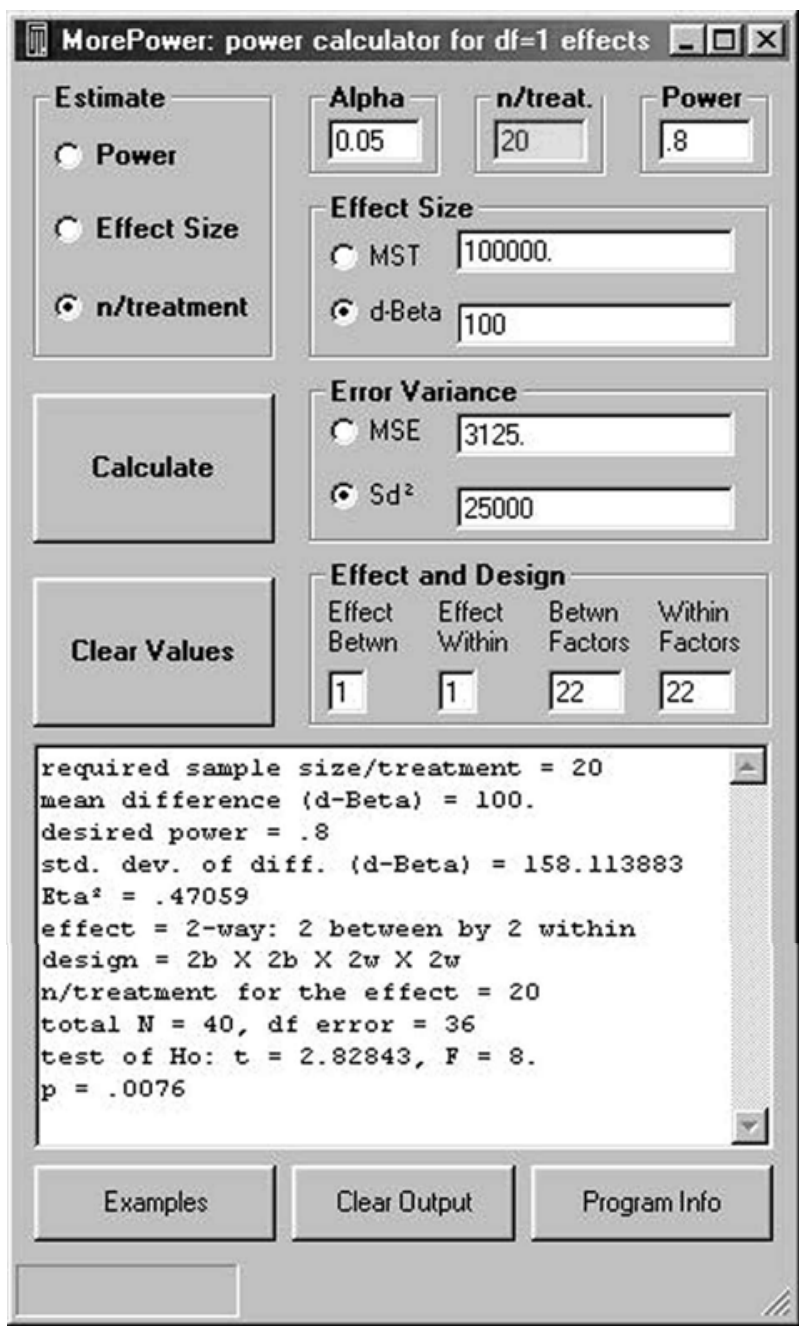

Figure 1. MorePower program interface.

ties, a prompt appears in the Output panel, and the cursor is moved to the appropriate data entry window on the interface. The desired estimate will appear in two places: (1) in the highlighted box on the interface (i.e., if one is calculating power, the value for power will appear in the upper right-hand box) and (2) in the Output window at the bottom of the calculator. As is illustrated in Figure 1, the Output window also displays other summary information for the analysis; this information may be copied and pasted into a word processor to be printed or saved.

To begin the next calculation, click the Clear Values button. At any point, one can change any quantity and recalculate. Positioning the mouse pointer over any label or data entry window on the MorePower interface displays a short description or instruction about the corresponding quantity.

The probability value entered or appearing in the Power panel of MorePower corresponds to the upper tail of the $t_{\beta}$ distribution (see Equation 3). The probability associ- 
ated with the lower tail usually is trivial from a practical standpoint; nonetheless, MorePower displays the exact power in the Output window, including both upper and lower tail probabilities. Probabilities are calculated using the BETAI algorithm for the incomplete beta function presented in Sprott (1996, p. 105).

There are 10 example sets of values for various between, within, and mixed effects that can be accessed by clicking the Test Example Values button on the lower left corner. Enter a number for the example one wishes to view and then click on the Calculate button.

\section{Examples}

Example 1: Estimating power and sample size for a $\boldsymbol{t}$ test. Suppose one measures reaction time (RT) for each of 25 people to make true/false decisions about a series of statements. True statements yield a mean of 1,000 msec, and false statements yield a mean of $1,050 \mathrm{msec}$. The standard deviation of the $50-\mathrm{msec}$ difference is $200 \mathrm{msec}$. To use the MorePower program to assess power, click Power in the upper left panel and press the Enter key on one's keyboard. Then enter 25 for $n_{\mathrm{t}}$ (n/treat on the MorePower interface), enter the mean difference of $50 \mathrm{msec}$ for $d_{\beta}$ (dBeta on the interface), and enter 40,000 (i.e., 2002) for $s_{d}^{2}$. To specify the effect and design (i.e., a paired-difference $t$ test), enter 1 for Effect Within (i.e., there is one twolevel factor in the effect of interest) and 2 for Within Factors (i.e., the design consists of two levels in one withinsubjects factor). The Effect Betwn and Betwn Factors boxes may be left blank or zeroes entered. Click on the Calculate button. The program displays an observed power of .21 for the 50 -msec difference, with $t(24)=1.25, p=.223$, for the two-tailed test of the null hypothesis.

To estimate the sample size required to detect a difference $\geq 50 \mathrm{msec}$ with a probability of .8 , click on $\mathrm{n} /$ treatment in the Estimate panel, which clears and highlights the $n /$ treat box. Press the Enter key (or point and click) to move the cursor to the Power input box, enter .8, and click on the Calculate button. The estimated sample size required (128) appears in the n/treat box and in the output window.

Example 2: Estimating power in multifactor designs. Consider a $2 \times 3 \times 3$ between-subjects design with 10 observations in each of the 18 cells $(N=180)$. The dependent measure is proportion of correct responses, and one wants to estimate the power to detect a main effect of .10 for the two-level factor. There are 90 observations contributing to each level of the main effect; thus, $n_{t}$ is 90 . One is interested in finding a difference of .10 between the two levels; thus, $d_{\beta}$ is .10 . For the main effect of the two-level factor, $F(1,162)=2.05, M S_{\mathrm{e}}=0.22, p>.05$.

In the MorePower program, select Power in the upper left panel and then enter $.05,90, .10$, and .22 in the data entry windows labeled Alpha, n/treatment, d-beta, and MSE, respectively. The effect of interest is a betweensubjects main effect (i.e., an effect involving a single factor), so enter 1 for Effect Betwn and either enter a 0 for
Effect Within or leave it blank. The complete design involves three between-subjects factors, and the values corresponding to the number of levels in each factor (i.e., 233, 323, or 332) should be entered for Betwn Factors. Within Factors should be set to 0 or left blank. Click on Calculate. The computed value for power should appear in the top right-hand corner, in the box labeled Power. Power for this example is computed to be .29, meaning that the probability is about $30 \%$ of finding a difference of $\geq .10$ between the two groups.

One can also use Equation 3 to perform the calculations by hand. Here, power is defined as the probability of finding a value of $t$ greater than $t_{\beta}$. We have included a table of $t$ values in the Appendix. If more precise values of the $t$ distribution are required, accurate probability calculators are available as stand-alone freeware (e.g., http://www.ncss.com/download.html).

To illustrate Equation 3, first, compute $s_{d}^{2}$ using Equation $7 \mathrm{a}$ :

$$
s_{d}^{2}=M S E \cdot 2^{B}=0.22 \cdot 2^{1}=0.44 .
$$

Substituting the remaining values into Equation 3, one gets

$$
t_{\beta}=1.98-\frac{.10}{\sqrt{\frac{.44}{90}}}=0.55 .
$$

From the Appendix, the probability that $t(162)$ exceeds $t_{\beta}$ is about .30 ; thus, the experiment had $30 \%$ power to detect a difference of .10 between levels of the main effect.

Example 3: Estimating effect size. Consider a $2 \times 2$ within-subjects experiment with an $N$ of 60 . The dependent variable is percentage correct, and one wishes to determine the size of the interaction that one could find with power equal to .8. For the interaction, $F(1,59)=191.36$, $M S E=27.15$.

To use MorePower, select Effect Size in the upper left box. Enter .05, 60, .80, and 27.15 in the boxes labeled Alpha, n/treatment, Power, and MSE, respectively. The effect of interest is a within-subjects interaction (i.e., an effect involving two factors), so enter 2 in the Effect Within box, and leave the Effect Betwn box blank. The complete design involves two within-subjects factors; enter 22 in the Within Factors box, and leave Betwn Factors empty. Click on Calculate. The computed value for $d_{\beta}$ should appear in the top Effect Size panel; this value is equal to 3.83, meaning that there is $80 \%$ power to find an interaction in which the difference of differences is equal to or greater than 3.83 .

One can also use Equation 4 to calculate effect size. For this equation, one needs $t_{\beta}$, which is the $t$ value associated with a given level of power (e.g., for power equal to .80 , one needs to find the value of $t$ above which .80 of the sampling distribution lies). In our example, with $d f$ equal to $59, t_{\beta}$ is -.849 (from the Appendix). For $\alpha$ at .05 , $t_{\alpha}=2.00$. 
Equation $7 \mathrm{~b}$ will be used to calculate $s_{d}^{2}$, where $W$ is the number of within-subjects factors associated with the relevant test (i.e., 2) and $y=$ the total number of cells in the design $(2 \times 2=4)$. Thus,

$$
s_{d}^{2}=27.15 \cdot \frac{2^{2 \cdot 2}}{4}=108.6 .
$$

Substituting these values into Equation 4:

$$
d_{\beta}^{2}=\sqrt{\frac{(2.00--.849)^{2} \cdot 108.6}{60}=3.8} .
$$

Example 4: Estimating sample size. Imagine planning a $2 \times 2 \times 2$ within-subjects RT experiment in which the three-way interaction is theoretically important. One wants to estimate the sample size required to detect a threeway effect (i.e., a difference between the difference of differences of $10 \mathrm{msec}$ or greater (i.e., $d_{\beta}=10$ ) with a power of .9. On the basis of previous research, we estimate $s_{d}^{2}$ for the three-way effect to be $50^{2} \mathrm{msec}$ (this can be estimated from the variance of a single cell, using Equation 7).

To use MorePower, select n/treatment in the upper left box. Enter .05, .90, 10, and 2500 in the boxes labeled Alpha, Power, d-beta, and $\mathrm{Sd}^{2}$, respectively. The effect of interest is a within-subjects interaction (i.e., an effect involving three factors), so enter 3 in the Effect Within box; leave the Effect Betwn box blank. The complete design involves three within-subjects factors; enter 222 in the Within Factors box, leaving Betwn Factors empty. Click on Calculate. The computed value for $n_{t}$ (i.e., 264) should appear in the top right-hand corner, in the box labeled $\mathrm{n} /$ treatment.

One can also use Equation 5 to calculate sample size. Again, one needs values of $t_{\alpha}$ and $t_{\beta}$; because these values depend upon sample size, Equation 5 may have to be solved iteratively. This will be an issue, however, only when one is concerned with small sample sizes, for which the values of $t_{\alpha}$ and $t_{\beta}$ may vary substantially as a function of $n_{\mathrm{t}}$. In this case, however, one can anticipate that $n$ will be quite large and, therefore, use the asymptotic values of $\mathrm{t}_{\alpha}(\infty)=1.960$ for $\alpha=.05$, and $\mathrm{t}_{\beta}(\infty)=-1.282$ for power $=.9$ (from the Appendix). Substituting into Equation 5 ,

$$
n_{\mathrm{t}}=\frac{(1.96--1.28)^{2} \cdot 50^{2}}{10^{2}} \approx 263 .
$$

\section{Conclusions}

Our approach simplifies several features of doing a power analysis. Specifying effect sizes is typically one of the most difficult features of doing a power analysis (Cohen, 1990, 1992). Effect sizes usually are expressed as a function of an estimate of error (e.g., Cohen, 1988; Howell, 1997) or in terms of a subjective evaluation of what constitutes a small, medium, or large effect size (Cohen, 1992). A convenient feature of our analysis is that it allows researchers to quantify effect sizes in terms of easy to understand, experimentally meaningful units. Similarly, the other quantities required are easily understood and accessible; researchers can enter values for $n_{\mathrm{t}}$, rather than having to compute $d f$, and use quantities such as $M S T$ and $M S E$ from an ANOVA to perform the calculations.

\section{REFERENCES}

Cochran, W. G., \& Cox, G. M. (1957). Experimental designs (2nd ed.). New York: Wiley.

CoHen, J. (1988). Statistical power analysis for the behavioural sciences. San Diego: Academic Press.

Cohen, J. (1990). Things I have learned (so far). American Psychologist, 45, 1304-1312.

Cohen, J. (1992). Quantitative methods in psychology. Psychological Bulletin, 112, 155-159.

HaYs, W. L. (1994). Statistics (5th ed.). Fort Worth, TX: Harcourt Brace. Howell, D. C. (1997). Statistical methods for psychology (4th ed.). Belmont, CA: Duxbury.

SpRotT, J. C. (1996). Numerical recipes and routines in BASIC. Cambridge, MA: Cambridge University Press.

Thomas, L., \& Krebs, C. J. (1997). A review of statistical power analysis software. Bulletin of the Ecological Society of America, 78, 126-139. 


\section{Power Levels and Corresponding $t$ Values}

Following are $t$ values associated with various power levels (i.e., upper-tail probabilities of the $t$ distribution). Note that the tabled values for tail probabilities greater than .5 (in bold) are negative. For example, to find the $t$ value associated with power equal to .8 and $d f$ equal to 30 , the tabled value would be -.854 . These values were calculated using a Hewlett-Packard 21S calculator.

\begin{tabular}{|c|c|c|c|c|c|c|c|c|c|c|}
\hline \multirow[b]{3}{*}{$d f$} & \multicolumn{10}{|c|}{ Power Levels } \\
\hline & .025 & .05 & .10 & .15 & .20 & .25 & .30 & .35 & .40 & .45 \\
\hline & .975 & .95 & .90 & .85 & .80 & .75 & .70 & .65 & .60 & .55 \\
\hline 1 & 12.706 & 6.314 & 3.078 & 1.963 & 1.376 & 1.000 & 0.727 & 0.510 & 0.325 & 0.158 \\
\hline 2 & 4.303 & 2.920 & 1.886 & 1.386 & 1.061 & 0.816 & 0.617 & 0.445 & 0.289 & 0.142 \\
\hline 3 & 3.182 & 2.353 & 1.638 & 1.250 & 0.978 & 0.765 & 0.584 & 0.424 & 0.277 & 0.137 \\
\hline 4 & 2.776 & 2.132 & 1.533 & 1.190 & 0.941 & 0.741 & 0.569 & 0.414 & 0.271 & 0.134 \\
\hline 5 & 2.571 & 2.015 & 1.476 & 1.156 & 0.920 & 0.727 & 0.559 & 0.408 & 0.267 & 0.132 \\
\hline 6 & 2.447 & 1.943 & 1.440 & 1.134 & 0.906 & 0.718 & 0.553 & 0.404 & 0.265 & 0.131 \\
\hline 7 & 2.365 & 1.895 & 1.415 & 1.119 & 0.896 & 0.711 & 0.549 & 0.402 & 0.263 & 0.130 \\
\hline 8 & 2.306 & 1.860 & 1.397 & 1.108 & 0.889 & 0.706 & 0.546 & 0.399 & 0.262 & 0.130 \\
\hline 9 & 2.262 & 1.833 & 1.383 & 1.100 & 0.883 & 0.703 & 0.543 & 0.398 & 0.261 & 0.129 \\
\hline 10 & 2.228 & 1.812 & 1.372 & 1.093 & 0.879 & 0.700 & 0.542 & 0.397 & 0.260 & 0.129 \\
\hline 11 & 2.201 & 1.796 & 1.363 & 1.088 & 0.876 & 0.697 & 0.540 & 0.396 & 0.260 & 0.129 \\
\hline 12 & 2.179 & 1.782 & 1.356 & 1.083 & 0.873 & 0.695 & 0.539 & 0.395 & 0.259 & 0.128 \\
\hline 13 & 2.160 & 1.771 & 1.350 & 1.079 & 0.870 & 0.694 & 0.538 & 0.394 & 0.259 & 0.128 \\
\hline 14 & 2.145 & 1.761 & 1.345 & 1.076 & 0.868 & 0.692 & 0.537 & 0.393 & 0.258 & 0.128 \\
\hline 15 & 2.131 & 1.753 & 1.341 & 1.074 & 0.866 & 0.691 & 0.536 & 0.393 & 0.258 & 0.128 \\
\hline 16 & 2.120 & 1.746 & 1.337 & 1.071 & 0.865 & 0.690 & 0.535 & 0.392 & 0.258 & 0.128 \\
\hline 17 & 2.110 & 1.740 & 1.333 & 1.069 & 0.863 & 0.689 & 0.534 & 0.392 & 0.257 & 0.128 \\
\hline 18 & 2.101 & 1.734 & 1.330 & 1.067 & 0.862 & 0.688 & 0.534 & 0.392 & 0.257 & 0.127 \\
\hline 19 & 2.093 & 1.729 & 1.328 & 1.066 & 0.861 & 0.688 & 0.533 & 0.391 & 0.257 & 0.127 \\
\hline 20 & 2.086 & 1.725 & 1.325 & 1.064 & 0.860 & 0.687 & 0.533 & 0.391 & 0.257 & 0.127 \\
\hline 21 & 2.080 & 1.721 & 1.323 & 1.063 & 0.859 & 0.686 & 0.532 & 0.391 & 0.257 & 0.127 \\
\hline 22 & 2.074 & 1.717 & 1.321 & 1.061 & 0.858 & 0.686 & 0.532 & 0.390 & 0.256 & 0.127 \\
\hline 23 & 2.069 & 1.714 & 1.319 & 1.060 & 0.858 & 0.685 & 0.532 & 0.390 & 0.256 & 0.127 \\
\hline 24 & 2.064 & 1.711 & 1.318 & 1.059 & 0.857 & 0.685 & 0.531 & 0.390 & 0.256 & 0.127 \\
\hline 25 & 2.060 & 1.708 & 1.316 & 1.058 & 0.856 & 0.684 & 0.531 & 0.390 & 0.256 & 0.127 \\
\hline 26 & 2.056 & 1.706 & 1.315 & 1.058 & 0.856 & 0.684 & 0.531 & 0.390 & 0.256 & 0.127 \\
\hline 27 & 2.052 & 1.703 & 1.314 & 1.057 & 0.855 & 0.684 & 0.531 & 0.389 & 0.256 & 0.127 \\
\hline 28 & 2.048 & 1.701 & 1.313 & 1.056 & 0.855 & 0.683 & 0.530 & 0.389 & 0.256 & 0.127 \\
\hline 29 & 2.045 & 1.699 & 1.311 & 1.055 & 0.854 & 0.683 & 0.530 & 0.389 & 0.256 & 0.127 \\
\hline 30 & 2.042 & 1.697 & 1.310 & 1.055 & 0.854 & 0.683 & 0.530 & 0.389 & 0.256 & 0.127 \\
\hline 40 & 2.021 & 1.684 & 1.303 & 1.050 & 0.851 & 0.681 & 0.529 & 0.388 & 0.255 & 0.126 \\
\hline 50 & 2.009 & 1.676 & 1.299 & 1.047 & 0.849 & 0.679 & 0.528 & 0.388 & 0.255 & 0.126 \\
\hline 100 & 1.984 & 1.660 & 1.290 & 1.042 & 0.845 & 0.677 & 0.526 & 0.386 & 0.254 & 0.126 \\
\hline$\infty$ & 1.960 & 1.645 & 1.282 & 1.036 & 0.842 & 0.674 & 0.525 & 0.386 & 0.254 & 0.126 \\
\hline
\end{tabular}

(Manuscript received October 16, 2000;

revision accepted for publication February 18, 2002.) 